\section{SCORERECALL: A Pascal program which scores protocols from multiple free-recall trials}

\author{
BURRTON WOODRUFF \\ Butler University, Indianapolis, Indiana
}

For a number of research paradigms in human learning, researchers ask that participants identify in free recall the stimuli previously presented. For some paradigms, such as those investigating hyperamnesia, participants may be asked to recall 40 or more items on multiple recall trials. The labels for the stimuli can differ from participant to participant as well as from trial to trial for any one participant. Scoring such protocols is a time-consuming, tedious, error-prone, boring task. After the protocols are scored, the results generally are entered on a computerreadable medium for statistical analysis.

The Program. SCORERECALL scores recall protocols and provides an output file which can be used as the input file of another program (an SPSSx program for example). Tables 1 through 6 document a short example showing the use of this program.

SCORERECALL uses as input two Pascal text files which can be created by clerical personnel with minimal training. The Thesaurus file contains the alternatives to be scored as correct responses for each stimulus itemthe alternatives for a stimulus are entered on one line and are separated by at least one blank. The Thesaurus file in Table 1 lists one to four correct responses for each of five stimulus items. As can be seen, it does not matter whether the alternatives are entered in upper or lower case; the number of leading and trailing spaces also is unimportant. The number of items, the number of alternatives per item, and the maximum length (number of characters) of each alternative are declared as global constants at the beginning of the program. In this example, SCORERECALL was compiled for five stimulus items, a maximum of three alternatives per stimulus item, and a maximum of 10 characters per alternative. In the example, the alternative "sailplane"' for Item 1 is ignored because it is a fourth alternative and only three are permitted by this compilation.

The other input file, the Dataln file, contains the responses of every participant on every recall trial. Each protocol begins with a record containing only a sentinel character that is declared as a global constant. An asterisk is used for the example in Table 2 . The second record is the identification code of that protocol. This code is

This work was done while the author was on a semester sabbatical provided by Butler University. The authors' mailing address is: Department of Psychology, Butler University, 4600 Sunset Avenue, Indianapolis, IN 46208.
Table 1

Example Thesaurus File for SCORERECALL Program

\begin{tabular}{lcc}
\hline $\begin{array}{l}\text { AIrplane } \\
\text { Lion }\end{array}$ & GLIDER & plane Sailplane \\
& CAstle & \\
Lemon & FRuIT & LIME \\
TeddyBear & BEAR & \\
\hline
\end{tabular}

Table 2

Example DataIn File for SCORERECALL Program

*

CN013

CASTLE

Lemon

Grape

*

CF023

Bear

castle

plane

camel

bear

*

CF021

Lion

plane

bear

*

CN012

CASTLE

BEAR

grapes

*

CF022

BEAR

LEMON

CASTLE

Plane

followed by the participant's responses-one response per line. In the example DataIn file, the identification code is four characters long, another global constant. The fifth character identifies the recall trial of the protocol. Recall trials are specified in the program by characters that are not necessarily integers. In Table 2 , recall trials have character codes between " 1 " and " 3 " which specify three recall trials per subject. Any two character codes separated by one other character in the system's collating sequence could have been used. Protocols do not have to be grouped by participant nor ordered by recall trial.

When SCORERECALL is executed, information about the scoring process is displayed at the terminal (Table 3 ). The operator sees a list of the alternative correct responses item by item; then, as each protocol is processed it is noted at the terminal. Finally a short message gives the names 
of the three output files and summarizes the contents of each.

The first output file, LogFile (Table 4), lists for each protocol the responses that could not be matched with any item from the Thesaurus file. The LogFile is useful in finding misspellings and typographical errors in both the Thesaurus and the DataIn files. The LogFile also indicates those participants who do not have all of their recall trials in the Dataln file. For the example, the LogFile notes that the Trial 1 protocol for subject CNO1 is not present in the input file.

The second output file, OutFile (Table 5), is designed to be the input for data analysis or statistical packages such as SPSSx. The first several lines of OutFile describe the contents of the data records. The data are ordered by subject and recall trial. The number of responses and the number of correct responses is given for each protocol in the input file. In addition, the OutFile includes how many correct responses were made for each item for each protocol.

The third output file, SubjRec, is a Pascal record file organized according to participants. For each trial, the

Table 3

Output to Terminal While SCORERECALL Processes the Example Data Set

\begin{tabular}{|c|c|c|}
\hline \multicolumn{3}{|c|}{ Alternative Correct Responses for Each Item } \\
\hline Item & Word & Alternative \\
\hline 1 & 1: & AIRPLANE \\
\hline 1 & 2: & GLIDER \\
\hline 1 & 3: & PLANE \\
\hline 2 & 1: & LION \\
\hline 2 & 2: & \\
\hline 2 & 3: & \\
\hline 3 & 1: & CASTLE \\
\hline 3 & 2: & \\
\hline 3 & 3: & \\
\hline 4 & 1: & LEMON \\
\hline 4 & 2: & |FRUIT \\
\hline 4 & 3: & LIME \\
\hline 5 & 1: & |TEDDYBEAR \\
\hline 5 & 2: & BEAR \\
\hline 5. & 3: & \\
\hline
\end{tabular}

Just Read Protocol

$\begin{array}{ll}\text { : CN01 } & \text { Trial: 3 } \\ \text { :CF02 } & \text { Trial: 3 } \\ \text { : CF02 } & \text { Trial: 1 } \\ \text { : CN01 } & \text { Trial: 2 } \\ \text { : CF02 } & \text { Trial: 2 }\end{array}$

Now writing OutFile.

Processing Completed. See LogFile for a listing of the responses that were NOT Correct for each Protocol. There is also a listing of any missing trials for any subject.

The SubjRec file is a Pascal record file of the number of Correct Responses, Number of Responses, and items that were correctly recalled. This information is given for each trial.

The SubjRec file can be further processed. The program RecRead prints them to the screen.

Outfile is a text file of subject data suitable for SPSSX processing It contains header information describing the contents of each line.
Table 4

Contents of the Example LogFile Written by SCORERECALL Program

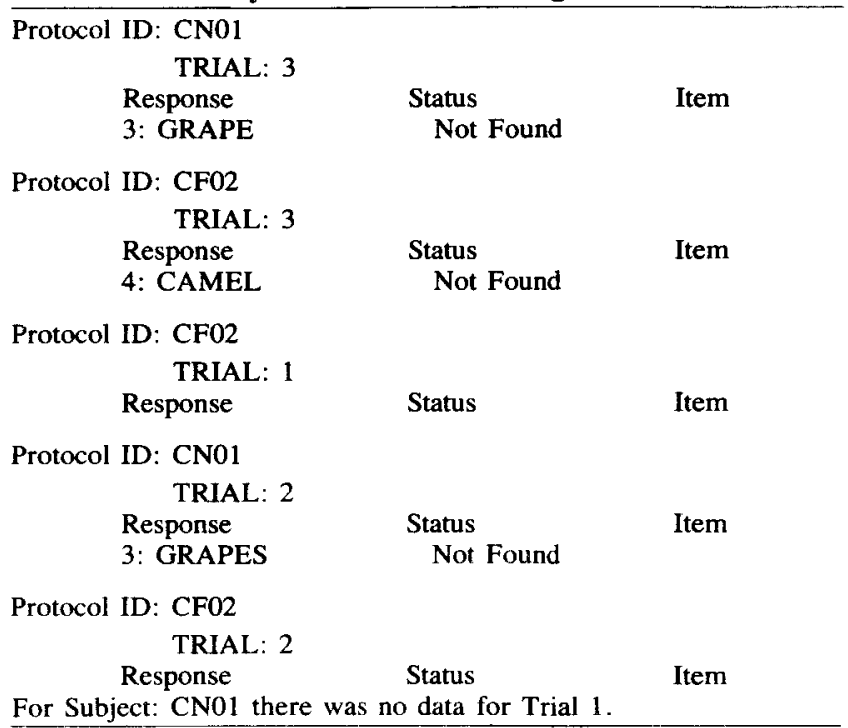

Table 5

Contents of Example OutFile Written by SCORERECALL Program

The data is output as sets of lines for each subject. The output for a subject is organized as pairs of lines for $\mathrm{EACH}$ recall trial

For a trial, line 1 contains:

IDString--The same as in the Dataln file.

Trial ID-- The same Character as in the DataIn File.

The Digit 1 to indicate a line 1 record.

Number of Responses.

Number of Correct Responses.

For a trial, line 2 contains:

IDString--The same as in the Dataln file.

Trial ID-- The same Character as in the Dataln File.

The Digit 2 to indicate a line 2 record.

A set of integers indicating the number of times the subject gave a correct response for that item. The ordering of integers is in terms of the ordering of the items in the Thesaurus file. The global Constant, ItemsPerLine, determines how many items will be output on line 2. Each succeeding line is in the same format as line 2 .

$\begin{array}{llllllll}\text { CNO12 } & 1 & & & & & 3 & 2 \\ \text { CNO12 } & 2 & 0 & 0 & 1 & 0 & 1 & \\ \text { CNO13 } & 1 & & & & & 3 & 2 \\ \text { CN013 } & 2 & 0 & 0 & 1 & 1 & 0 & \\ \text { CF021 } & 1 & & & & & 3 & 3 \\ \text { CF021 } & 2 & 1 & 1 & 0 & 0 & 1 & \\ \text { CF022 } & 1 & & & & & 4 & 4 \\ \text { CFO22 } & 2 & 1 & 0 & 1 & 1 & 1 & \\ \text { CF023 } & 1 & & & & & 4 & 3 \\ \text { CF023 } & 2 & 1 & 0 & 1 & 0 & 1 & \end{array}$

file contains a list of the responses, the number of responses, the number of correct responses, and the number of times the participant correctly responded to each stimulus item. Table 6 shows the contents of the SubjRec 
Table 6

Partial Listing of the Output of the RECREAD Program Which Displays the Contents of the Example SubjRec File Produced by SCORERECALL

Subject Identification: CNO1

Trial Number: 1

No. Responses: -1 No. Correct: 0

$\begin{array}{cc}\text { Item } & \text { No. Responses } \\ 1 & 0 \\ 2 & 0 \\ 3 & 0 \\ 4 & 0 \\ 5 & 0 \\ \text { Item } & \text { Response }\end{array}$

Trial Number: 2

No. Responses: 3 No. Correct: 2

\begin{tabular}{cc} 
Item & No. Responses \\
1 & 0 \\
2 & 0 \\
3 & 1 \\
4 & 0 \\
5 & 1 \\
Item & Response \\
1 & CASTLE \\
2 & LEMON \\
3 & GRAPE \\
\hline
\end{tabular}

file for two trials of one subject as listed to the terminal by a short utility program (RECREAD). As can be seen, the missing protocol for this subject is indicated by showing -1 responses for Trial 1 . For Trial 2, the subject's three responses are given. The SubjRec file facilitates further data analysis since Pascal record files are more easily accessed and processed than are text files created with an editor.

Program Requirements. The program is written in standard Pascal for the VAX 11/780 under the VMS operating system.

Program Availability. The 20-page printout of the extensively documented source code for SCORERECALL and RECREAD is available free of charge from the author. The source code is also available on magnetic tape to those who send a tape and return postage.

(Revision accepted for publication April 23, 1985.) 